\title{
A Woman With Massive Splenomegaly
}

\author{
Erin Meschter, MSIII, Michelle Choi, MD, and Lisa Teng, MD
}

\section{Case Presentation}

An 82 year-old Caucasian woman was admitted to the hospital with marked splenomegaly and pancytopenia. Her primary care physician noted splenomegaly on physical exam during a recent visit, and a CT scan of the abdomen was performed as an outpatient which revealed a massively enlarged spleen. The patient admitted to losing up to 40 pounds over the past six months due to decreased appetite and early satiety. She also reported dyspepsia and a feeling of fullness in her belly for several months. The patient denied nausea, vomiting, constipation, or diarrhea. Other symptoms included increased fatigue, chronically swollen legs, and frequent night sweats two to three nights per week with occasional fevers. The patient denied shortness of breath, cough, or chest pain. She complained of urinary urgency and frequency.

The patient had a past medical history of hypertension and osteoarthritis in her knees and ankles. Her past surgical history included cataract surgery and cholecystectomy. Family history was significant for her mother's "heart problems" and her father dying from pneumonia. The patient had never smoked, although she frequently experienced second hand smoke. She did not drink, nor did she have a history of alcohol abuse. She never used any illegal drugs or recreational substances. Medications on admission included Clonidine $0.1 \mathrm{mg}$ BID and hydrocholorothiazide $12.5 \mathrm{mg}$ every day. She had no known drug allergies.

On physical exam her temperature was $97.8^{\circ}$ Fahrenheit, pulse was 98 beats/minute, and respiration rate was 19 breaths/ minute. Her blood pressure was 129/71 mm Hg, and her oxygen saturation was $98 \%$ while breathing ambient air. The patient was in no apparent distress and was alert and oriented to time, place, and self. She was slender with a protuberant abdomen. The spleen was palpable beyond the umbilicus into the right upper quadrant. She had no lymphadenopathy. She was pale, but not jaundiced, and had no apparent ascites. She had a 2/6 systolic ejection murmur with no heave and a normal PMI. Her lungs were clear to auscultation bilaterally. She had numbness in her index and ring finger on both hands and some left-sided facial numbness in the distribution of the $\mathrm{V} 2$ branch of cranial nerve five. Neurologic exam revealed no other abnormalities. She had no joint swelling or erythema, but her legs had pitting edema bilaterally.

On admission, chest radiograph and CT of the abdomen with contrast showed massive splenomegaly $(24.5$ X 15.1 X $11.7 \mathrm{~cm}$, see Figure 1). Her CBC revealed pancytopenia with a white blood cell count of $2.4 \mathrm{~B} / \mathrm{L}$, red blood cells $3.36 \mathrm{~B} / \mathrm{L}$, platelets $94 \mathrm{~B} / \mathrm{L}$, hemoglobin $10.6 \mathrm{~g} / \mathrm{dL}$, and hematocrit $32.8 \%$. Reticulocyte count was elevated at $2.1 \%$. Of note, she had an elevated lymphocyte count of 45.5; neutrophils were low at 36.4. Electrolytes were within normal limits. Albumin was low at $2.9 \mathrm{~g} / \mathrm{dL}$ and $\mathrm{LDH}$ was normal at $199 \mathrm{U} / \mathrm{L}$. Her blood smear showed pancytopenia consistent with her complete blood count along with poikilocytosis and suspected villous lymphocytes. Vitamin B12 was very low at $52 \mathrm{pmol} / \mathrm{L}$. A Monospot test was non-reactive.

A bone marrow biopsy was performed a few days after admission along with flow cytometry. The bone marrow biopsy indicated patches of hypercellularity with lymphoid aggregates consisting of monotonous, small round cells without cytoplasm suggestive of small lymphocytes. Paratrabecular aggregates also were appreciated. The bone marrow had maintained good hematopoietic activity. Of note, reticulin staining of the bone marrow was increased in the aggregates indicating that this bone marrow was more difficult to aspirate and thus aggregate cells may have been less represented on smear. Markers were used to stain the bone marrow. A marker for CD3 was used to stain $\mathrm{T}$ cells, which were sprinkled throughout the bone marrow with a few in the lymphoid aggregates. A marker for CD20 was used to stain B cells, which were scattered throughout the marrow. Lymphoid aggregates were predominantly CD20 positive, suggestive of a B cell lymphoproliferative disorder.

Flow cytometry was used to distinguish what type of lymphoproliferative disorder was present in this woman's bone marrow. Total lymphocytes were $35 \%$, which is slightly elevated. Of the lymphocytes, $31 \%$ were B cells ( $15 \%$ to $20 \%$ is normal). The B cells present were CD10 negative, which eliminated follicular cell lymphoma from the differential. The $\mathrm{B}$ cells were CD5 negative, making Mantle cell lymphoma and chronic lymphocytic leukemia (CLL) less likely. There were some B cells that were CD11c positive, a marker for Hairy cell leukemia; but a more specific marker for Hairy cell is CD103, which was negative in this patient's bone marrow biopsy. Most revealing was the kappa and lambda Ig marker staining, which is used to evaluate for clonality. The flow cytometry showed kappa restricted B cell clonality (see Figure 2). Through a process of elimination via this analysis, a diagnosis of splenic marginal zone lymphoma (SMZL) was suspected. There are no distinguishing markers for SMZL, and this type of lymphoma shares many markers with other lymphoproliferative diseases. It is interesting to note that there is CD15 positivity in the $\mathrm{B}$ cells present in this patient, and perhaps this marker should be followed in future diagnoses of SMZL to discover a new correlation.

\section{Discussion}

Splenic marginal zone lymphoma (SMZL) is an indolent B-cell malignancy involving the spleen, bone marrow, and blood. It is a rare lymphoma type, first described in 1992, and has been estimated as less than $2 \%$ of all patients with lymphoma. ${ }^{2}$

Splenic marginal zone lymphoma is a disease of the elderly with a median age at presentation of 65 years old. ${ }^{2}$ Almost all patients 
present with moderate to massive splenomegaly, sometimes with abdominal discomfort. Hepatomegaly can sometimes be observed, but lymphadenopathy is uncommon. Anemia and thrombocytopenia are common at presentation secondary to hypersplenism more than bone marrow infiltration. Autoimmune phenomena, such as primary biliary cirrhosis, rheumatoid arthritis, immune thrombocytopenia, are present in $10 \%$ to $20 \%$ of patients. ${ }^{2,4}$ Splenic marginal zone lymphoma is considered a low-grade lymphoma with an indolent clinical course, and median survival is close to ten years, with five-year survival estimated to be greater than $65 \%{ }^{1}$ Approximately $10 \%$ of patients undergo transformation to a high-grade lymphoma.

The diagnosis is based on lymphocyte morphology, immunophenotype, bone marrow histology, cytogenetic abnormalities, and when available, spleen histology. Approximately 75\% of patients with splenic marginal zone lymphoma will have a peripheral blood lymphocytosis. ${ }^{2}$ One of the most characteristic findings is the presence of villous lymphocytes on peripheral blood smear, which are intermediate-sized lymphocytes with condensed chromatin and short cytoplasmic projections. ${ }^{2}$ However, the amount of circulating cells may be so small, that it cannot be detected. Infiltration of the bone marrow is evident in virtually all patients with SMZL, but is invariably involved. It may be demonstrable only by flow cytometry and not by morphology alone. The immunophenotype profile for SMZL is typically positive for CD20, CD45RA, CD45RB, CD79A, PAX5/ BSAP, IgM, and bcl2, and negative for CD43, CD23, CD10, bc16, and cyclin D1. ${ }^{1}$

The pattern of bone marrow infiltration can vary and may be nodular, intransinusoidal, interstitial, and paratrabecular. ${ }^{4}$ However, intrasinusoidal infiltration is considered to be characteristic of SMZL. In SMZL, the spleen is enlarged and splenic sections demonstrate a nodular replacement of white pulp, usually with a central core of lymphocytes with slightly irregular nuclei, and an outer zone of larger cells with clear cytoplasm in the marginal zone. Both cell populations are part of the neoplastic clone. The splenic red pulp is also involved in most cases, usually occupying germinal centers and is evident by immunostaining. ${ }^{5}$ Although a primary diagnosis of SMZL is rarely, if ever, made on the basis of lymph node biopsy, splenic hilar nodes are typically involved. Complex chromosomal aberrations are common in SMZL; $80 \%$ of cases have an abnormal karyotype, but there is no single abnormality present in all cases. ${ }^{2}$ The most frequent cytogenetic aberrations are gains of $3 q$ and $12 q$ and $7 q$ deletion. ${ }^{2}$

There is no definitive standard treatment for splenic marginal zone lymphoma to date. The level of evidence supporting recommendations for initiating treatment is weak due to the absence of prospective clinical trials. Currently treatment is based on clinical evidence obtained from clinical centers with experience in treating SMZL. Due to the indolent course of
SMZL, about two thirds of patients are asymptomatic at diagnosis and as many as one third will never require therapy. In general, the development of lymphadenopathy, high lymphocyte count, or infiltration of non-hematopoietic sites have been found to be associated with progression and shorter overall survival. ${ }^{2}$ Progression may also be associated with a transformation to a high grade lymphoma.

However, therapeutic intervention should be considered in patients with active disease. Considering the indolent course of SMZL, the patient's age and comorbidities, treatment should be aimed at achieving control of the disease and symptoms rather than eradication of the disease. ${ }^{2}$ There are several therapeutic options effective in these patients including splenectomy, chemotherapy, and irradiation. Symptomatic splenomegaly or severe cytopenia are the main indications to perform splenectomy. However, splenectomy alone cannot reduce the extrasplenic lymphomatous infiltrations, and one group has reported an increase of tumor burden in the bone marrow after splenectomy. ${ }^{1}$ Splenic irradiation has been used in a limited number of patients and has been reported to produce a reduction in circulating villous lymphocytes, regression of splenomegaly, and improvement of cytopenias. ${ }^{1}$ Chemotherapy is generally used as a first line treatment in people with more advanced disease. Alkylating agents appear to be of marginal benefit. Purine analogs, such as fludarabine in combination with rituximab, or rituximab alone, have demonstrated a greater efficacy than alkylating agents in terms of response and progression-free survival. ${ }^{2}$

Our patient's clinical presentation and findings were consistent with splenic marginal zone lymphoma. She received splenic irradiation and underwent splenectomy for symptomatic splenomegaly. She is currently undergoing chemotherapy with Rituxan.

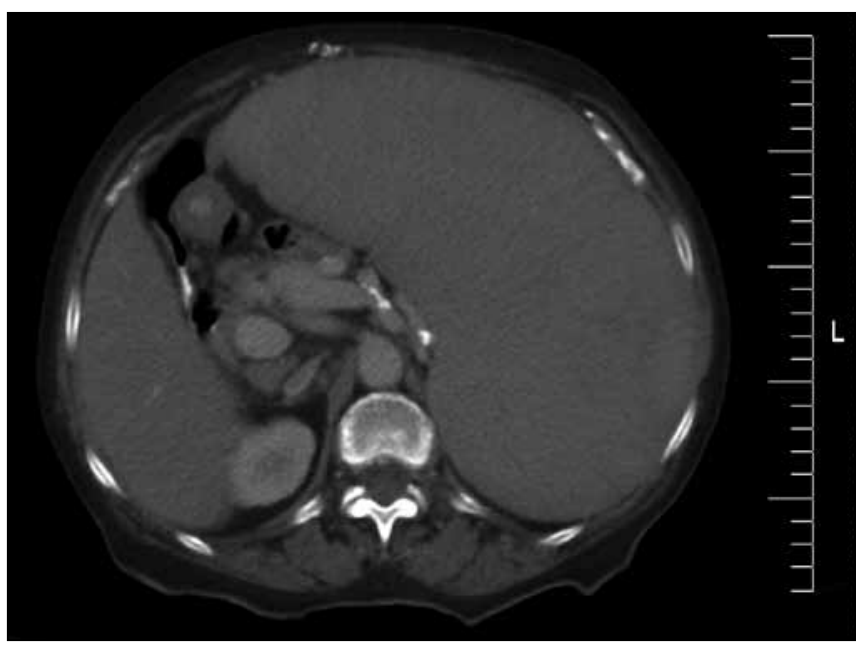

Figure 1. On CT of the abdomen with contrast, the spleen was found to be massively enlarged measuring at least 24.5 X $15.1 \mathrm{X}$ $11.7 \mathrm{~cm}$ and is homogenously enhancing. 

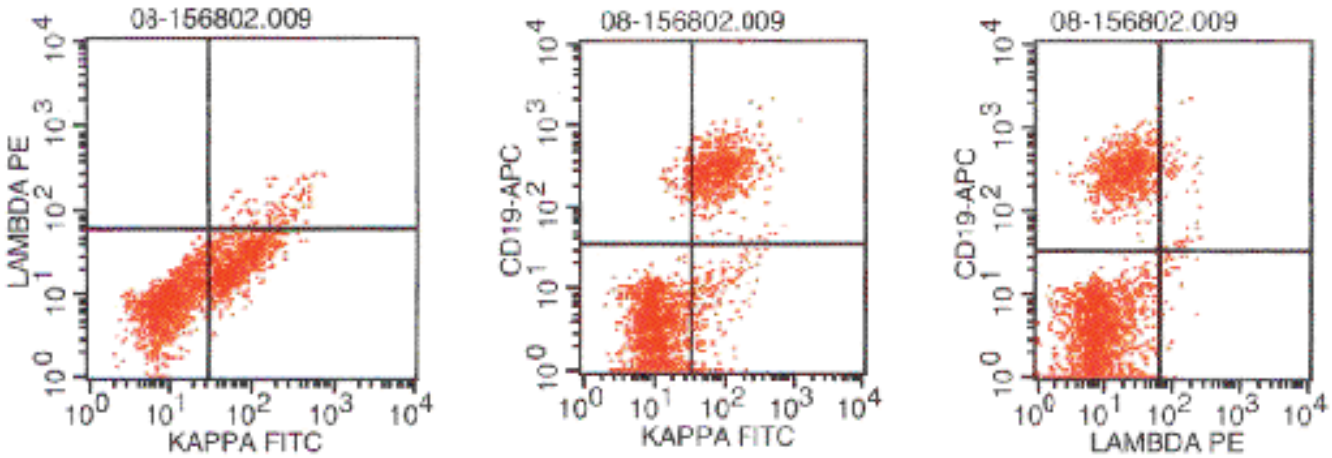

\begin{tabular}{crrr} 
Quad & Events & $\%$ Gated $\%$ Total \\
\hline UL & 0 & 0.00 & 0.00 \\
UR & 113 & 4.14 & 1.13 \\
LL. & 1780 & 65.27 & 17.80 \\
LF & 834 & 30.58 & 8.34
\end{tabular}

\begin{tabular}{crrr} 
Quad & Events & $\%$ Gated & $\%$ Total \\
\hline UL & 60 & 2.20 & 0.60 \\
UH & 702 & 25.74 & 7.02 \\
LL & 1774 & 65.05 & 17.74 \\
LR & 191 & 7.00 & 1.91
\end{tabular}

\begin{tabular}{rrrr} 
Quad & Everts & Gated $\%$ Total \\
\hline UL & 706 & 25.89 & 7.06 \\
UR & 58 & 2.13 & 0.58 \\
LL & 1936 & 70.59 & 19.36 \\
LR & 27 & 0.59 & 0.27
\end{tabular}

Figure 2. All three flow cytometry graphs show kappa-restricted B cell clonality, suggestive of a B cell lymphoproliferative disease. Graph A shows only kappa positive marker; the same cells are negative for lambda marker. CD19 is a marker for B cells, and is utilized in Graph B and C. Graph $B$ displays CD19 positive cells are also kappa positive, but they are lambda negative (in Graph C).

\section{References}

1. Franco V. Florena AM. Iannitto E. Splenic marginal zone lymphoma. Blood. 101(7):2464-72, 2003 Apr 1.

2. Matutes E. Oscier D. Montalban C. Berger F. Callet-Bauchu E. Dogan A. Felman P. Franco V. Iannitto E. Mollejo M. Papadaki T. Remstein ED. Salar A. Sole F. Stamatopoulos K. Thieblemont C. Traverse-Glehen A. Wotherspoon A. Coiffier B. Piris MA. Splenic marginal zone lymphoma proposals for a revision of diagnostic, staging and therapeutic criteria. Leukemia. 22(3):487-95, 2008 Mar.

3. Mollejo M. Camacho FI. Algara P. Ruiz-Ballesteros E. Garcia JF. Piris MA. Nodal and splenic marginal zone B cell lymphomas. Hematological Oncology. 23(34):108-18, 2005 Sep-Dec.

4. Oscier D. Owen R. Johnson S. Splenic marginal zone lymphoma. Blood Reviews. 19(1):39-51, 2005 Jan.

5. Thieblemont C. Felman P. Callet-Bauchu E. Traverse-Glehen A. Salles G. Berger F. Coiffier B. Splenic marginal-zone lymphoma: a distinct clinical and pathological entity. Lancet Oncology. 4(2):95-103, 2003 Feb. 